\title{
Metformin and Exercise the Effects of Metformin in the Body Building to Minimize the Effects of the use of Anabolic: Case Report for a Randomized Controlled Trial
}

\author{
Khaled Hamlaoui* and Dario Furnari \\ Department of Biomedical Sciences, Hospital, Netherland, United Kingdom \\ *Corresponding author: Khaled Hamlaoui, Department of Biomedical Sciences, Hospital, Netherland, United Kingdom
}

\begin{abstract}
Metformin is an oral antidiabetic used in the treatment of type 2 diabetes mellitus. More precisely, it belongs to the class of biguanides, Metformin is used in the treatment of type 2 diabetes mellitus both as monotherapy and in combination therapy with other oral antidiabetic agents or with insulin, when dietary interventions and exercise are not sufficient to control the disease. When used in overweight diabetic patients, metformin also causes a decrease in the complications of diabetes and its use has been associated with stabilization and, albeit modest, loss of body weight. In type 2 diabetes mellitus (called also DM2 and in the past 'adult diabetes' or 'food') the insulin produced by the pancreas is unable to fully exert its action so that the body even produces it in excess, with the result on the one hand of making increasing weight and on the other hand progressively depleting the pancreas, is unable to meet the body's needs. It is as if the body was resisting the action of insulin. Metformin reduces insulin resistance. It is taken by mouth and is the only drug useful in all stages of type 2 diabetes. It also helps improve the balance of fats and, to a limited extent, blood pressure.
\end{abstract}

Metformin alone has important effects on blood sugar. Accompanied by physical exercise, weight loss and possibly other medications, it is often an effective therapy. It does not cause hypoglycemia, helps not to gain weight or even reduces it. The main feature of Metformin is to interact strongly with AMPK by regulating its expression. In fact, its downregulation leads to consuming ATP, synthesizing cholesterol and fatty acids and consuming glucose: a situation in which insulin levels are quite high (therefore energy abundance). On the contrary, its upregulation leads to the creation of ATP, consuming more fatty acids for energy purposes and it is a metabolic situation similar to caloric restriction in which insulin levels are kept low (therefore energy scarcity). Metformin by upregulating AMPK has therefore shown to have a somewhat transversal therapeutic use in the treatment of metabolic dysfunc

Keywords: Exercise; Nutritional Biochemistry; Metformin; Body Building; Inflammation; Skeletal Muscle

\section{Introduction}

The use of anabolic androgenic steroids (AAS) in bodybuilding became a huge problem and risky factor that causes the death of many athletes, extreme ways are followed in bodybuilding especially during contest preparation and the goal is to get the perfect physique on stage, our goal is to help and save lives by providing scientific evidence to help the athletes and save their lives by preventing them the abuse of such drugs, in our case report series we used Metformin. Within the cell, Metformin inhibits the inflammatory pathway and activates AMPK (thereby increasing mTOR inhibition), while also modulating oxidative stress. These processes jointly affect inflammation, cellular survival, stress defense, autophagy, and protein synthesis. Metformin can increase insulin sensitivity and lower blood levels of insulin due to its ability to cleanse the microbiome, also inhibiting glucose formation in the liver, can also inhibit the release of pro-inflammatory cytokines, reduces or eliminates inflammatory factors.Metformin increases the formation of mucin-degrading Akkermansia miciniphila in the gut. Akkermansia muciniphila reduces inflammation, lowers insulin resistance so by this we can say simply that metformin boosts the growth of good bacteria. Metformin is effective and efficient drug 
for our case report series, because of the following mechanisms of action:

\section{Insulin \& Inflammation Suppression}

- Neuroprotection

- Anti-cancer agent

- microbiome cleansing

- $\quad$ Akkermansia producing

\section{Body Fat Burning}

It is interesting to note that relatively recent studies suggest that Metformin can directly inhibit the action of leucine on mTOR. Not only would this be a bad sign for muscle growth, but the inhibitory effect of Metformin on mTOR should have a major effect as it correlates with the reduction in the risk of fatal cancers in diabetics. Interesting as postulated by Dr. Melnik of the University of Osnabrück in Germany: Metformin may be a direct competitor of leucine for the binding and activity of mTORC1. The doctor noted in his article that the usual daily dose in diabetics of Metformin ( $2 \mathrm{~g}$ ) is in the range of the $2 \mathrm{~g}$ of leucine derived from the daily consumption of $100 \mathrm{~g}$ of meat or cheese. Since the two substances are similar in structure and size, they can compete for the same sites in the activation of mTOR. However, Metformin has these three characteristics of relevant interest:

\section{Schematic Representation of Gluconeogenesis}

- Increases the number and sensitivity of cell receptors for Insulin.

- Decreases the amount of glucose / sugar absorbed by the intestine.

- It decreases the amount of glucose / sugar produced by the liver (a primary source for the production of hepatic glucose is amino acids). These three effects explain why clinical administration of Metformin rarely results in cases of greater hypoglycemic effects when administering the drug alone. However, when given in combination with insulin and / or sulfonylureas (Glipizide) injections there is a significant increase in carbohydrate requirements and weight gain. The aforementioned characteristics expressed by Metformin have meant that this drug enters the arsenal of sports pharmacological supplementation, especially in Body Building.

\section{Metformin and Body Builders}

Generally, Body Builders use the effects of Metformin differently for different phases:

- During the Bulk phases, with the use of exogenous insulin, 500-800mg of Metformin 1-2 times a day increased the effectiveness of the insulin. This was due to an increase in the number and sensitivity of the receptor sites. Metformin also decreases the amount of insulin required for maximum results.

- During pancreatic regeneration or protocols containing Glipizide, Glyburide or other pancreatic stimulants / insulin, Metformin increases its effectiveness and amplifies the results. The common dose of Metformin for this purpose is $500 \mathrm{mg} 2$ times a day.

- $\quad$ During the Cut phases, Body Builders use Metformin as a means of decreasing the production of glucose by the liver and the absorption of glucose by the intestine. By itself, this decreases the secretion of insulin by the pancreas and increases the body's dependence on fat stores for energy needs. This is especially done while using GH and PGF-2 and creates a synergistic effect with AAS. As the cellular receptor sites for insulin are more sensitive and since there is cross stimulation between IGF-1 and insulin (and their opposite receptor sites) the retention of lean mass increases contained. This effect favors the reduction of the negative effects that a weight loss diet exerts on the endogenous production of IGF-1: if a coadministration of IGF-1 and Metformin occurs, the advantage reflects on the lower dose (of IGF-1) required and on the better receptor efficiency.

It is known that during a calorie-deficient diet the IGF-1 produced decreases and is one of the factors by which the stored lean mass is reduced. If the cell sites are more numerous and sensitive, stimulation requires less IGF-1. Typically, 500mg of Metformin per day is considered sufficient. Metformin should be taken with meals and never less than six hours before going to bed. People with kidney problems should not take Metformin and most athletes should be aware that in some cases the combination with 17-alpha-alkylated drugs can induce even greater liver damage.

\section{Experimental Procedure}

Design, Setting, Participants

\section{Methods}

The purpose of this case report series is to evaluate the potential interaction between metformin and exercise during contest prep in order to help in different contest protocols to minimize the use of extreme ways used during preparations, and save the health of bodybuilders.

Table 1: 6 Bodybuilders with no Chronic Disease and on Very Healthy Diet.

\begin{tabular}{|c|c|}
\hline Condition & Intervention/Treatment \\
\hline Contest Prep: 5 Weeks Out & Drug: Metformin \\
\hline & $\begin{array}{c}\text { Dosage: } 1500 \mathrm{mg} / \text { Day (First } 2 \\
\text { Weeks) }\end{array}$ \\
\hline Participants: 6 Bodybuilders. & $2000 \mathrm{mg} /$ Day (Last 2 Weeks) \\
\hline Age: $20-34$ Years & $0 \mathrm{Mg}$ (During Peak Week) \\
\hline
\end{tabular}


The method used to measure cortisol levels: The cobas e 411 fully automated, system for immunoassay analysis

We increased the essential amino acids intake and GH doses (for AAS users) during the last two weeks, and every bodybuilder ingested $7 \mathrm{~g}$ of glutamine during working out.

During contest prep the most important thing is to get the best look lean and dry BUT without putting the athlete's life on risk of any health issues.

The key thing of this experiment was to not mess with minerals balance in the body, we know certain anabolic steroids cause minerals imbalance so we focused on that and tried using metformin during the prep and we collected blood samples from the participants every week.

The other thing $\mathrm{i}$ focused on was cortisol levels and inflammatory biomarkers and metformin have a direct antiinflammatory action. Studies have suggested that metformin suppresses inflammatory response by inhibition of nuclear factor $\kappa \mathrm{B}(\mathrm{NF \kappa B})$ via $\mathrm{AMP}$-activated protein kinase (AMPK)-dependent and independent pathways.

We noticed an improve in gut health and no digestion issue (no bloating) and was just one case had diarhea.

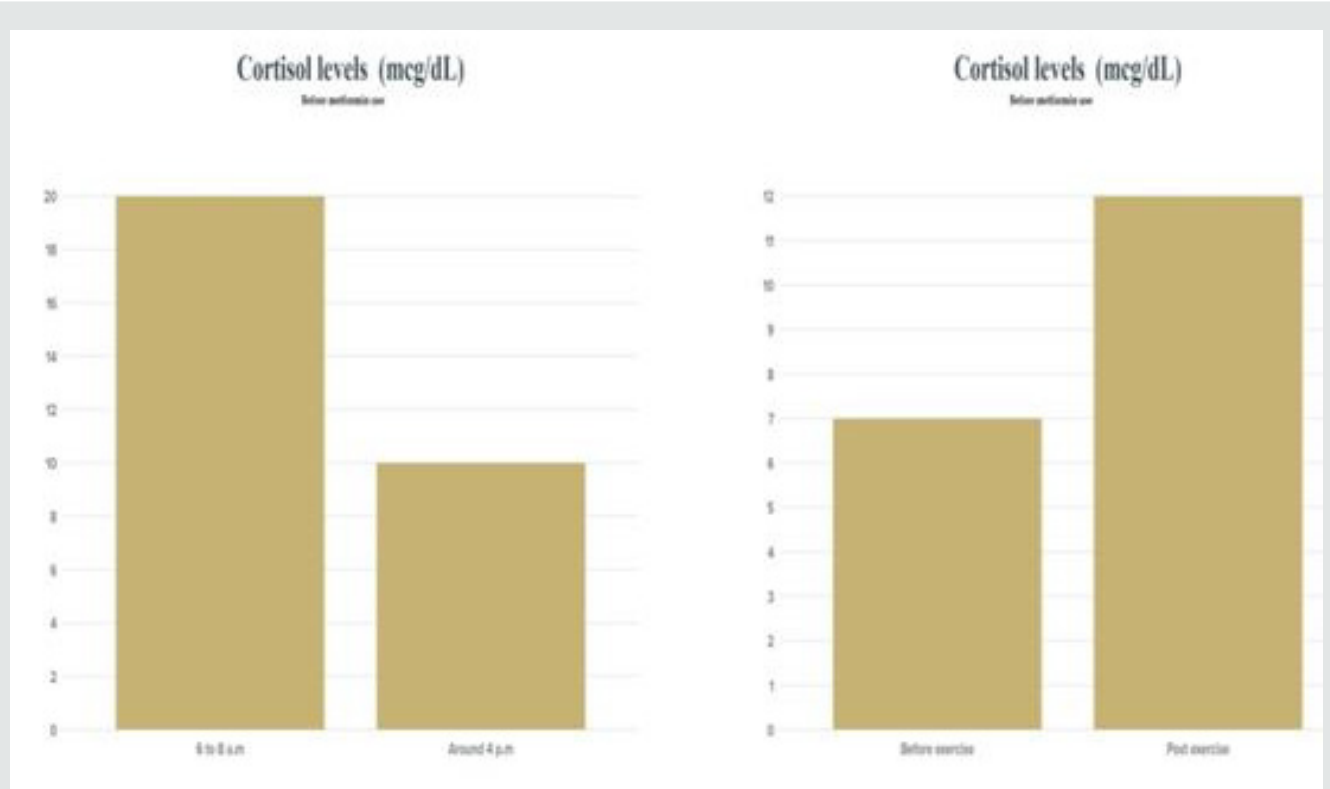

Figure 1: Cortisol levels before the use of Metformin . The data were collected a week before the experiment daily.

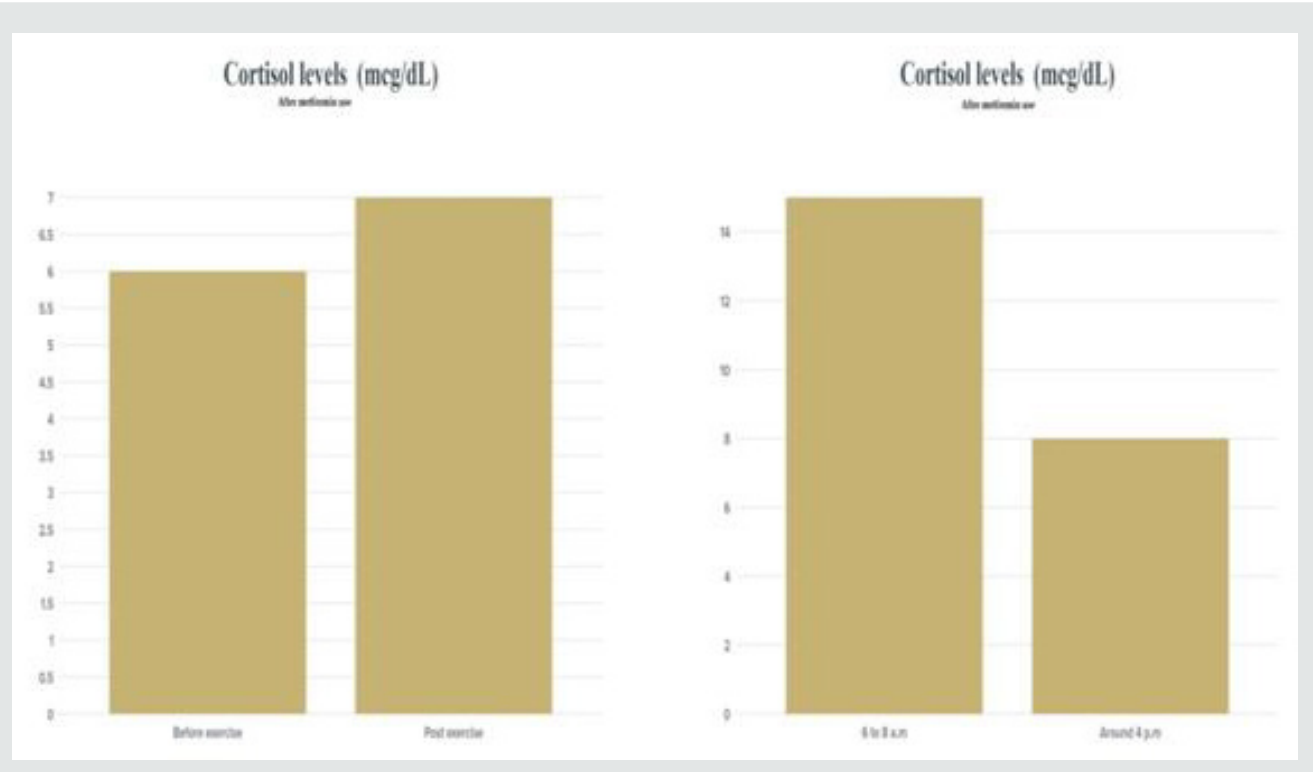

Figure 2: Cortisol levels after the use of Metformin. 


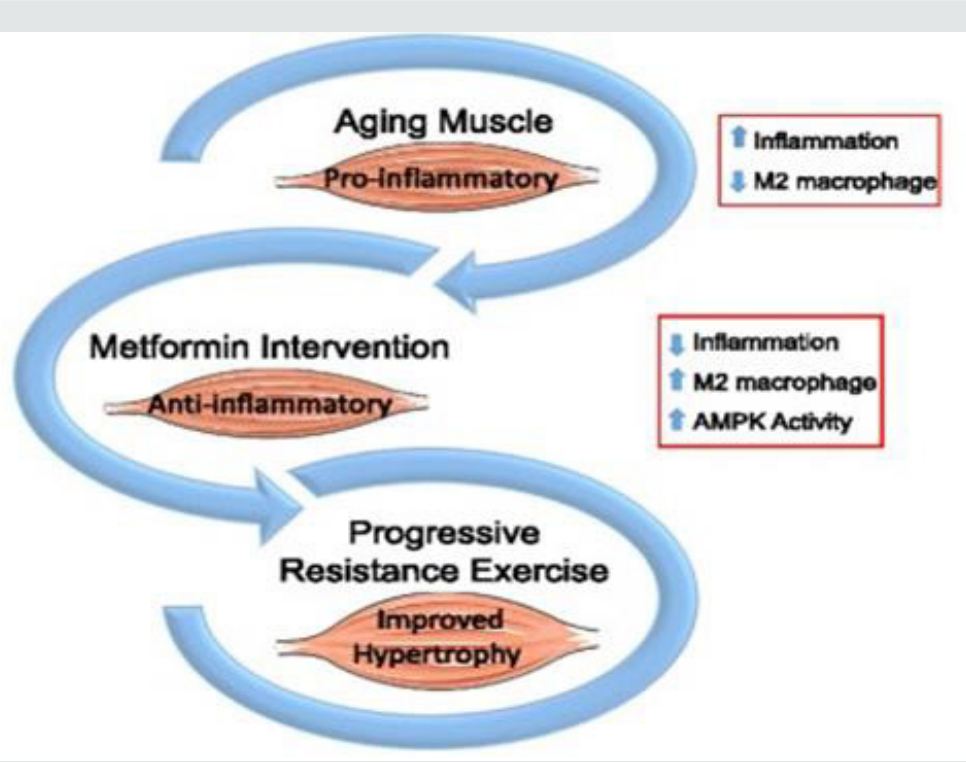

Figure 3.

\section{Discussion}

Therefore Metformin (by upregulating AMPK) implements:

- Reduction of insulin secretion

- Reduction of the synthesis of fatty acids and cholesterol from the two previous situations derives:

- increased lipolysis

- increased lipid oxidation, uptake of glucose and glycolysis in skeletal muscles

- Increase in lipid oxidation and uptake of glucose by the heart

- Inhibition of pro-inflammatory processes

- Reduction of glucose absorption from the gastrointestinal tract to about half

- Inhibition of hepatic gluconeogenesis by $36 \%$

- Increase in the number and sensitivity of insulin receptors,

- increasing the uptake of glucose on peripheral structures

- increases lipid oxidation for energy purposes

From the following meta-analysis it was found that metformin reduced by:

- $5.3 \%$ the BMI and triglycerides

- 4.5\% fasting blood sugar

- $14.4 \%$ fasting insulin

- 5.6\% LDL cholesterol

- $40 \%$ the onset of diabetes (in pre-diabetic subjects)
Increasing HDL cholesterol by 5\%. Therefore, metformin could also be used in dyslipidemic subjects with moderately altered LDL values, instead of using statins, and in association with repaglinide as it has shown on the one hand to increase insulin sensitivity and on the other to improve the lipid profile.

However, the mechanism by which it manages to activate AMPK is currently unclear, but other hypotheses on how Metformin can inhibit the hepatic production of glucose have been advanced. For example, it has been hypothesized that metformin may inhibit mitochondrial glycerol 3-phosphate dehydrogenase (mGPD) which would reduce the production of hepatic glucose from glycerol and lactate, but the shuttle system of glycerol phosphate is of little relevance.

Hepatic unlike that of aspartate malate, so much so that on an animal model the destruction of the shuttle glycerol phosphate did not alter glycaemia and it could also be expected that gluconeogenesis from lactate would not be affected since the NADH produced by the conversion of lactate pyruvate would have been consumed by 3-phosphate glyceraldehydes

\section{Dehydrogenase During Gluconeogenesis}

However, a link was found between these two mechanisms, as it was found that the upregulation of AMPK inhibited mGPD in yeasts (very similar to that of humans), this would explain the possible interaction between the pathway that regulates MGPD and the direct action that Metformin exerts on AMPK.

\section{Conclusion}

Metformin one of the most studied drugs, based on the collected data, we found it's suitable for our case report series, because of its anti-aging and anti-inflammatory properties, it helped the athletes 
to benefit and minimize the risks, and our main goal is to save many lives possible.

Something similar might be going on with metformin and exercise. ROS, cytokines, and myokines, for example, are involved in the adaptation process. If metformin is blunting these signals, it's interfering with the adaptation. The perceived stress in the body may be artificially attenuated by the drug and therefore the compensatory response is, too. I will save you from all of the gory details in this email, but I may elaborate on the mechanisms and biochemistry down the road.

So, if you're a relatively healthy person that exercises regularly and takes metformin as an anti-aging drug, should you continue taking metformin? I don't think there's a black and white answer at this point and it depends on how you personally respond. In the most recent study, there was a high amount of variability in the metformin group in their response to exercise-induced adaptations. For me, in response to these papers, along with my personal experience of seeing slightly higher lactate levels during zone 2 training(suggesting, perhaps, less mitochondrial efficiency), I've reduced my dose of metformin and only take it in the evening in an effort to reduce the amount of metformin in my system when I'm awake and exercising.

Maybe there's an optimal dosing, timing, and scheduling of metformin, fasting, nutritional makeup, and exercise that's superior to placebo. Maybe not. And it depends on your makeup and your individual response.

\section{References}

1. Shaw JE, Sicree RA, Zimmet PZ (2010) Global estimates of the prevalence of diabetes for 2010 and 2030. Diabetes Research and Clinical Practice 87(1): 4-14.

2. Xu Y, Wang L, He J, Yufang Bi, Mian Li, et al. (2013) Prevalence and control of diabetes in Chinese adults. JAMA 310(9): 948-959.

3. Chatterjee S, Khunti K, Davies MJ (2017) Type 2 diabetes. The Lancet 389(10085): 2239-2251.

4. Bragg F, Holmes MV, Iona A, Yu Guo, Huaidong Du, et al. (2019) Association between diabetes and cause-specific mortality in rural and urban areas of China," JAMA 317(3): 280-289.

5. Foretz M, Guigas B, Bertrand L, Pollak M, Viollet B, et al. (2014) Metformin: from mechanisms of action to therapies. Cell Metabolism 20(6): 953-966.

6. American Diabetes Association (2016) 7 Approaches to glycemic treatment. Diabetes Care 39(Supplement 1): S52-S59.

7. Nathan DM, Buse JB, Davidson MB, Ele Ferrannini, Holman RR, et al. (2009) Medical management of hyperglycaemia in type 2 diabetes mellitus: a consensus algorithm for the initiation and adjustment of therapy: a consensus statement from the American Diabetes Association and the European Association for the Study of Diabetes. Diabetologia 32(1): 193-203.

8. Hundal RS, Krssak M, Dufour S, D Laurent, V Lebon, et al. (2000) Mechanism by which metformin reduces glucose production in type 2 diabetes. Diabetes 49(12): 2063-2069.
9. An H, He L (2000) Current understanding of metformin effect on the control of hyperglycemia in diabetes. Journal of Endocrinology 228(3): R97-R106.

10. Greco M, Chiefari E, Montalcini T, Francesca Accattato, Francesco S Costanzo, et al. (2014) Early effects of a hypocaloric, Mediterranean diet on laboratory parameters in obese individuals. Mediators of Inflammation 2014: 750860.

11. Mensink M, Blaak EE, Wagenmakers AJ, Saris WH (2005) Lifestyle intervention and fatty acid metabolism in glucose-intolerant subjects Obesity Research 13(8): 1354-1362.

12. Goodyear LJ, Kahn BB (1998) Exercise, glucose transport, and insulin sensitivity. Annual Review of Medicine 49(1): 235-261.

13. Koivisto VA, Yki-Järvinen H, DeFronzo RA (1986) Physical training and insulin sensitivity Diabetes/Metabolism Reviews 1(4): 445-481.

14. CO'Hagan, G De Vito, Boreham CAG (2013) Exercise prescription in the treatment of type 2 diabetes mellitus: current practices, existing guidelines and future directions Sports Medicine 43(1): 39-49.

15. Church TS, Blair SN, Cocreham S, Neil Johannsen, William Johnson, et al. (2010) Effects of aerobic and resistance training on hemoglobin A1C levels in patients with type 2 diabetes: a randomized controlled trial. JAMA 304(20): 2253-2262.

16. Sharoff CG, Hagobian TA, Malin SK, Stuart R Chipkin, Haiyan Yu, et al. (2010) Combining short-term metformin treatment and one bout of exercise does not increase insulin action in insulin-resistant individuals American Journal of Physiology-Endocrinology and Metabolism 298(4): E815-E823.

17. Malin SK, Gerber R, Chipkin SR, Braun B (2012) Independent and combined effects of exercise training and metformin on insulin sensitivity in individuals with prediabetes. Diabetes Care 35(1): 131136.

18. Malin SK, Nightingale J, Choi SE, Chipkin SR, Braun B (2013) Metformin modifies the exercise training effects on risk factors for cardiovascular disease in impaired glucose tolerant adults. Obesity 21(1): 93-100.

19. Boule NG, Kenny, Larose J, Khandwala F, Kuzik N, et al. (2013) Does metformin modify the effect on glycaemic control of aerobic exercise, resistance exercise or both?" Diabetologia, 56(11): 2378-2382.

20. Hansen M, Palsoe MK, Helge JW, Dela F (2015) The effect of metformin on glucose homeostasis during moderate exercise. Diabetes Care 38(2): 293-301.

21. Erickson ML, Little JP, Gay JL, McCully KK, Jenkins NT (2017) Postmeal exercise blunts postprandial glucose excursions in people on metformin monotherapy. Journal of Applied Physiology 123(2): 444-450.

22. Chacko E (2017) A time for exercise: the exercise window," Journal of Applied Physiology, 122(1): 206-209.

23. Macfarlane DJ, Y Lee CC, EY K Ho, Chan KL, DTS Chan, et al. (2007) Reliability and validity of the Chinese version of IPAQ (short, last 7 days)," Journal of Science and Medicine in Sport, 10(1): 45-51.

24. Kuipers H, Verstappen FT, Keizer HA, Geurten P, G van Kranenburg, et al. (1985) Variability of aerobic performance in the laboratory and its physiologic correlates. International Journal of Sports Medicine 6(4): 197-201.

25. Revdal A, Hollekim-Strand SM, Ingul CB (2016) Can time efficient exercise improve cardiometabolic risk factors in type 2 diabetes? A pilot study. J Sports Sci Med 15(2): 308-313.

26. Robinson E, Durrer C, Simtchouk S, Mary E Jung, Jessica E, Bourne et al. (2015) Short-term high-intensity interval and moderate-intensity continuous training reduce leukocyte TLR4 in inactive adults at elevated risk of type 2 diabetes. Journal of Applied Physiology 119(5): 508-516. 
27. Horton TJ, Grunwald GK, Lavely J, Donahoo WT (2006) Glucose kinetics differ between women and men, during and after exercise. Journal of Applied Physiology 100(6): 1883-1894.

28. Weston KS, Wisloff U, Coombes JS (2014) High-intensity interval training in patients with lifestyle-induced cardiometabolic disease: a systematic review and meta-analysis. British Journal of Sports Medicine 48(16): 1227-1234.

29. Batacan Jr RB, Duncan MJ, Dalbo VJ, Tucker PS, Fenning AS (2017) Effects of high-intensity interval training on cardiometabolic health: a systematic review and meta-analysis of intervention studies. British Journal of Sports Medicine 51(6): 494-503.

30. Cassidy S, Thoma C, Houghton D, Trenell MI (2017) High-intensity interval training: a review of its impact on glucose control and cardiometabolic health. Diabetologia 60(1): 7-23.

31. Gillen JB, Little JP, Punthakee Z, Tarnopolsky MA, Riddell MC, Gibala M (2012) Acute high-intensity interval exercise reduces the postprandial glucose response and prevalence of hyperglycaemia in patients with type 2 diabetes," Diabetes, Obesity and Metabolism 14(6): 575-577.

32. Karstoft K, Christensen CS, Pedersen BK, Solomon TPJ (2014)The acute effects of interval- vs continuous-walking exercise on glycemic control in subjects with type 2 diabetes: a crossover, controlled study. The Journal of Clinical Endocrinology \& Metabolism, vol. 99(9): 3334-3342.

33. Frontoni S, Bartolo P Di, Avogaro A, Bosi E, Paolisso G, et al. (2013) Glucose variability: an emerging target for the treatment of diabetes mellitus Diabetes Research and Clinical Practice 102(2): 86-95.

34. Monnier L, Mas E, Ginet C, Françoise Michel, Laetitia Villon, et al. (2006) Activation of oxidative stress by acute glucose fluctuations compared with sustained chronic hyperglycemia in patients with type 2 diabetes. JAMA 295(14): 1681-1687.

35. Rizzo MR, Marfella R, Barbieri M, Virginia Boccardi, Francesco Vestini, et al. (2010) Relationships between daily acute glucose fluctuations and cognitive performance among aged type 2 diabetic patients. Diabetes Care 33(10): 2169-2174

36. Torimoto K, Okada Y, Mori H, Tanaka Y (2013) Relationship between fluctuations in glucose levels measured by continuous glucose monitoring and vascular endothelial dysfunction in type 2 diabetes mellitus," Cardiovascular Diabetology 12(1): 1

37. Boule NG, Robert C, Bell GJ, Steven T Johnson, Rhonda C Bell, et al. (2011) Metformin and exercise in type 2 diabetes: examining treatment modality interactions. Diabetes Care 34(7): 1469-1474.

38. Gopaul NK, Anggard EE, Mallet AI, Betteridge DJ, Wolff SP, et al. (1995) Plasma 8-epi-PGF2 $\alpha$ levels are elevated in individuals with non-insulin dependent diabetes mellitus. FEBS Letters 368(2): 225-229.

39. Meigs JB, Larson MG, Fox CS, Keaney JF, Vasan RS, et al. (2007) Association of oxidative stress, insulin resistance, and diabetes risk phenotypes: the Framingham Offspring Study. Diabetes Care 30(10): 2529-2535.

40. Radak Z, Zhao Z, Koltai E, Ohno H, Atalay M, et al. (2013) Oxygen consumption and usage during physical exercise: the balance between oxidative stress and ROS-dependent adaptive signaling. Antioxidants \& Redox Signaling 18(10): 1208-1246.

41. Malin SK, Braun B (2016) Impact of metformin on exercise-induced metabolic adaptations to lower type 2 diabetes risk. Exercise and Sport Sciences Reviews 44(1): 4-11.

42. McArdle A, Pattwell D, Vasilaki A, Griffiths RD, Jackson MJ, et al. (2001) Contractile activity-induced oxidative stress: cellular origin and adaptive responses," American Journal of Physiology-Cell Physiology, vol. 280(3): C621-C627.

43. Sampson MJ, Gopaul N, Davies IR, Hughes DA, Carrier MJ, et al. (2002) Plasma F2 isoprostanes: direct evidence of increased free radical damage during acute hyperglycemia in type 2 diabetes," Diabetes Care 25(3): 537-541.

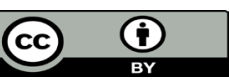

This work is licensed under Creative Commons Attribution 4.0 License

To Submit Your Article Click Here: Submit Article

DOI: $10.32474 /$ OAJOM.2020.04.000180

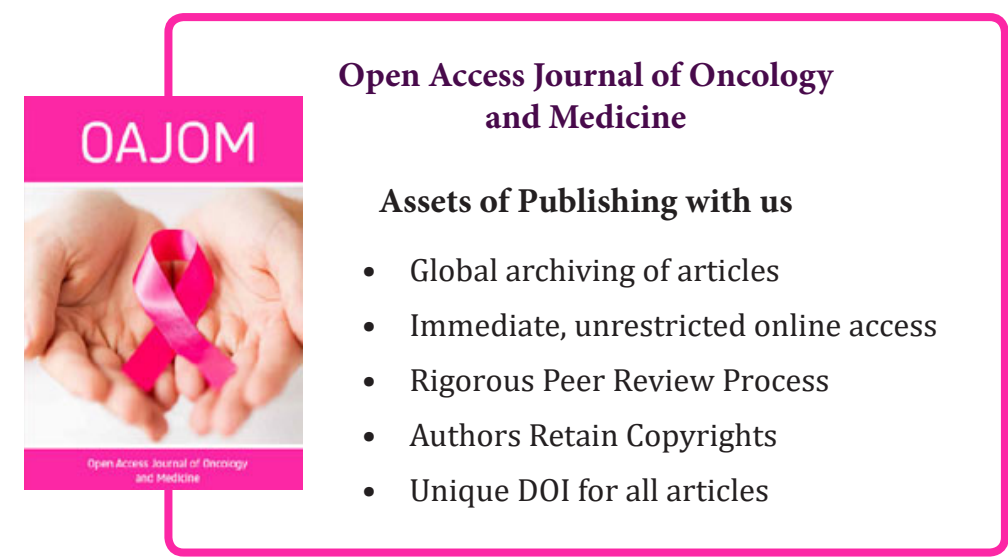

